\title{
Perioperative anxiety: A short review
}

\section{Dr. Shailendra Sigdel*}

Assistant Professor, Department of Anesthesiology, Tribhuvan University Manmohan Cardiothoracic Vascular and Transplant Center, Institute of Medicine, Maharahgunj, Kathmandu, Nepal

\section{Introduction}

Anxiety is the subjective unpleasant feelings of dread over something unlikely to happen, such as the feeling of imminent death. It is often accompanied by restlessness, fatigue, problems in concentration, and muscular tension. Perioperative anxiety is described as a vague, uneasy feeling, the source of which is often nonspecific and unknown to the individual [1] but known to cause abnormal hemodynamics as a consequence of sympathetic, parasympathetic and endocrine stimulation. Anxiety occurs in any person in a transient or chronic form and can produce aggressive reactions that result in increased stress experienced by the patient, thus causing more difficult pain management in the postoperative period [2]. Perioperative period is a stressful event that triggers specific emotional, cognitive, and physiological responses of a patient.

The incidence of preoperative anxiety varies according to the setting of surgery, gender and motives for surgery. The prevalence is higher ranging from $32 \%$ in a study done on patients awaiting general surgery [3] to $50 \%$ in patients awaiting coronary artery bypass graft surgery (CABG) [4] and the prevalence is similar or even higher in Asian population [5].

\section{Factors related to perioperative anxiety}

Factors responsible for preoperative fears depend on age, gender, single or divorce, education, uncertainty of the exact day of surgery, patient's ability to understand the events that occur during surgical anesthesia, fear of surgery, separation from their family, financial loss, postoperative pain, fear of death and fear of unknown origin [6-8]. Lack of adequate and timely information to patients during the preanesthetic consultation increases patient anxiety. Study by Kiyohara et al. [9] found that patients receiving better preanesthetic information during the visit with the anesthesiologist showed reduced rates of anxiety compared to those who did not receive it. The day of admission can also be very stressful, as patients have to cope with both the stress of hospitalization and the anxiety about the impending surgery.

\section{Psychological response to perioperative anxiety}

The extent of anxiety levels varies individually. It fluctuates over time; starting prior to the surgery and persists until the late postoperative period. Different patient react perioperative periods in different ways. Some find it as relief as they are going to have a disease free life. Other considered it as one of the stressful event of lifetime. They are preoccupied with their discomfort or concerned about the success of surgery, strong fear of failure combined with career and family problems, postoperative state of physical health and problems adapting to the changed situation.
The consequences of perioperative anxiety are major cardiac events [10-13] (acute myocardial infarction, heart failure, pulmonary edema), high readmission rate ( $1^{\text {st }} 6$ month, 1 years), $[10,11]$ poor quality of life and high rate of cardiac mortality. Impact correlate with high postoperative pain, increased analgesic and anesthetic consumption, prolonged hospital stay, adverse influence during anesthetic induction and patient recovery and decrease patient satisfaction with perioperative experience.

The reasons of increased morbidity in anxious patient are associated with the development of cardiovascular lesions as a consequences of health-related behaviors [14] (such as smoking, poor diet, poor compliance with treatment, or an inactive lifestyle) and direct influence on the myocardial perfusion, autonomic nervous system regulation, platelet activation, increased hypothalamo-pituitary-adrenal axis activity and exaggerated inflammatory processes [14-16].

Preoperative anxiety level is difficult to measure accurately. However, it can be estimated indirectly by measuring blood pressure, pulse,and decreased heart rate variability and patient irritability. Directly, it can also be estimated by measuring the plasma of cortisol and urinary level of catecholamine. At present, several validated questionnaires [5] are available and used to measure preoperative anxiety. These include Amsterdam Preoperative Anxiety Information Scale (APAIS), the State Trait Anxiety Inventory (STAI), Hospital Anxiety and Depression Scale (HADS), Visual Analogue Scale (VAS), Multiple Affect Adjective Check List (MAACL). The APAIS is a widely accepted [5] screening tool which has been translated and used in many countries including Germany, the Netherlands, Mexico, Thailand, Turkey Korea and Japan.

\section{Management}

These patients need to be intervened before and after surgery to reduce the morbidity and mortality. Interventions before surgery include developing good rapports and doctor patient relationships, education and structured interviews, psychotherapy, selective serotonin reuptake inhibitors (SSRIs) and benzodiazepine. The routine evaluation and effectively addressing the preoperative psychological distress facilitate early postoperative recovery. Early intervention in postoperative period to patients with evidence of psychological distress offers reduction of hospital length of stay, analgesic use, postsurgical

Correspondence to: Dr. Shailendra Sigdel, Assistant Professor, Department of Anesthesiology, Tribhuvan University Manmohan Cardiothoracic Vascular and Transplant Center, Institute of Medicine, Maharahgunj, Kathmandu, Nepal, Tel: +97-798-511-23474; E-mail: sigdelshailendra@gmail.com

Received: July 04, 2015; Accepted: August 14, 2015; Published: August 17, 2015 
morbidity and help patients to adopt more effective coping strategies in their everyday lives.

\section{Conclusions}

There is prevalence of high degrees of preoperative anxiety in patients with elective surgery. Perioperative anxiety is often overlooked but it is associated with poor surgical outcome. At present several validated questionnaires are available to measure preoperative anxiety. Preoperative counseling and proper education regarding surgery will help in reducing preoperative anxiety and improving the quality of care.

\section{References}

1. Klopfenstein CE, Forster A, Van Gessel E (2000) Anesthetic assessment in an outpatient consultation clinic reduces preoperative anxiety. Can J Anaesth 47: 511515. [Crossref]

2. Anderson KO, Masur FT 3rd (1983) Psychological preparation for invasive medical and dental procedures. J Behav Med 6: 1-40. [Crossref]

3. Moerman N, van Dam FS, Muller MJ, Oosting H (1996) The Amsterdam Preoperative Anxiety and Information Scale (APAIS). Anesth Analg 82: 445-451. [Crossref]

4. Koivula M, Paunonen-Ilmonen M, Tarkka MT, Tarkka M, Laippala P (2001) Fear and anxiety in patients awaiting coronary artery bypass grafting. Heart Lung 30: 302-311. [Crossref]

5. Matthias AT, Samarasekera DN (2012) Preoperative anxiety in surgical patients experience of a single unit. Acta Anaesthesiol Taiwan 50: 3-6. [Crossref]

6. Thomas V, Heath M, Rose D, Flory P (1995) Psychological characteristics and the effectiveness of patient-controlled analgesia. Br J Anaesth 74: 271-276. [Crossref]
7. Caumo W, Schmidt AP, Schneider CN, Bergmann J, Iwamoto CW, et al. (2001) Risk factors for postoperative anxiety in adults. Anaesthesia 56: 720-728. [Crossref]

8. Sukantarat KT, Williamson RC, Brett SJ (2007) Psychological assessment of ICU survivors: a comparison between the Hospital Anxiety and Depression scale and the Depression, Anxiety and Stress scale. Anaesthesia 62: 239-243. [Crossref]

9. Kiyohara LY, Kayano LK, Oliveira LM, Yamamoto MU, Inagaki MM, et al. (2004) Surgery information reduces anxiety in the pre-operative period. Rev Hosp Clin Fac Med Sao Paulo 59: 51-56. [Crossref]

10. Saur CD, Granger BB, Muhlbaier LH, Forman LM, McKenzie RJ, et al. (2001) Depressive symptoms and outcome of coronary artery bypass grafting. Am J Crit Care 10: 4-10. [Crossref]

11. Scheier MF, Matthews KA, Owens JF, Schulz R, Bridges MW, et al. (1999) Optimism and rehospitalization after coronary artery bypass graft surgery. Arch Intern Med 159: 829-835. [Crossref]

12. Baker RA, Andrew MJ, Schrader G, Knight JL (2001) Preoperative depression and mortality in coronary artery bypass surgery: preliminary findings. ANZ J Surg 71: 139-142. [Crossref]

13. Perski A, Feleke E, Anderson G, Samad BA, Westerlund H, et al. (1998) Emotional distress before coronary bypass grafting limits the benefits of surgery. Am Heart $J 136$ : 510-517. [Crossref]

14. Rozanski A, Blumenthal JA, Kaplan J (1999) Impact of psychological factors on the pathogenesis of cardiovascular disease and implications for therapy. Circulation 99: 2192-2217. [Crossref]

15. Musselman DL, Evans DL, Nemeroff CB (1998) The relationship of depression to cardiovascular disease: epidemiology, biology, and treatment. Arch Gen Psychiatry 55: 580-592. [Crossref]

16. Kubzansky LD, Kawachi I, Weiss ST, Sparrow D (1998) Anxiety and coronary heart disease: a synthesis of epidemiological, psychological, and experimental evidence. Ann Behav Med 20: 47-58. [Crossref]

Copyright: (C2015 Sigdel S. This is an open-access article distributed under the terms of the Creative Commons Attribution License, which permits unrestricted use, distribution, and reproduction in any medium, provided the original author and source are credited. 